\title{
TonEBP in dendritic cells mediates pro- inflammatory maturation and Th1/Th17 responses
}

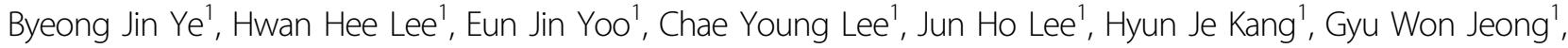 \\ Hyun Park', Whaseon Lee-Kwon', Soo Youn Choi ${ }^{1}{ }^{1}$ and Hyug Moo Kwon'
}

\begin{abstract}
Dendritic cells (DCs) are potent antigen-presenting cells that link the innate and adaptive immune responses; as such they play pivotal roles in initiation and progression of rheumatoid arthritis (RA). Here, we report that the tonicityresponsive enhancer-binding protein (TonEBP or NFAT5), a Rel family protein involved in the pathogenesis of autoimmune disease and inflammation, is required for maturation and function of DCs. Myeloid cell-specific TonEBP deletion reduces disease severity in a murine model of collagen-induced arthritis; it also inhibits maturation of DCs and differentiation of pathogenic Th1 and Th17 cells in vivo. Upon stimulation by TLR4, TonEBP promotes surface expression of major histocompatibility complex class II and co-stimulatory molecules via p38 mitogen-activated protein kinase. This is followed by DC-mediated differentiation of pro-inflammatory Th1 and Th17 cells. Taken together, these findings provide mechanistic basis for the pathogenic role of TonEBP in RA and possibly other autoimmune diseases.
\end{abstract}

\section{Introduction}

Dendritic cells (DCs) are professional antigenpresenting cells (APCs) that are uniquely capable of priming naïve $\mathrm{T}$-cells, although macrophages and B-cells are also able to process and present antigens to $\mathrm{T}$-cells ${ }^{1}$. The maturation process, which upregulates expression of MHC class II and co-stimulatory molecules at the cell surface, is central to DC function ${ }^{2}$. Mature DCs migrate to draining lymph nodes (LNs) and promote differentiation of naive T-cells into effector T-cells; they also activate various other immune cells depending on the stimuli that they sense in $\mathrm{LNs}^{2}$. Accordingly, evidence from clinical studies and experimental models implicates DCs in the pathogenesis of most autoimmune diseases, including multiple sclerosis, systemic lupus erythematosus, and rheumatoid arthritis (RA) ${ }^{3}$.

\footnotetext{
Correspondence: Soo Youn Choi (sychoi@unist.ac.kr) or

Hyug Moo Kwon (hmkwon@unist.ac.kr)

'School of Life Sciences, Ulsan National Institute of Science and Technology, Ulsan 44919, Republic of Korea
}

Edited by Y. Shi
RA, one of the most prevalent autoimmune diseases in humans, is characterized by chronic inflammation and destruction of bone and cartilage within joints, leading ultimately to chronic pain, severe disability, and increased mortality ${ }^{4}$. The diagnosis and management of early arthritis are pivotal to prevent damage from becoming clinically significant ${ }^{5}$. Because dysregulation of immune cells plays a critical role in RA pathogenesis, its reversal is a major therapeutic goal ${ }^{6,7}$. Although effective therapies are available, a significant number of RA patients do not respond well ${ }^{8}$. Accordingly, better understanding of the causative and disease-contributing factors is needed. DCs are emerging as an important cell population that plays a role in RA pathogenesis ${ }^{9-11}$. DCs are involved in initiating the disease via production of cytokines and presentation of arthritogenic antigens, which together activate autoreactive Th1 and Th17 cells; it is the latter that actually cause the damage associated with $\mathrm{RA}^{9,10}$. In addition, DCs contribute to the marked increase in leukocyte infiltration into synovial tissue ${ }^{11}$. Data from human patients and murine models of early RA show that DC numbers in joint-draining LNs increase prior to visible histological

\section{(c) The Author(s) 2020}

(c) Open Access This article is licensed under a Creative Commons Attribution 4.0 International License, which permits use, sharing, adaptation, distribution and reproduction cc) in any medium or format, as long as you give appropriate credit to the original author(s) and the source, provide a link to the Creative Commons license, and indicate if changes were made. The images or other third party material in this article are included in the article's Creative Commons license, unless indicated otherwise in a credit line to the material. If material is not included in the article's Creative Commons license and your intended use is not permitted by statutory regulation or exceeds the permitted use, you will need to obtain permission directly from the copyright holder. To view a copy of this license, visit http://creativecommons.org/licenses/by/4.0/. 
changes, demonstrating the importance of DCs in initiating $\mathrm{RA}^{12}$. Furthermore, reduced numbers of circulating DCs in patients with RA correlate with an enriched synovial population of DCs possessing a high T-cell stimulatory capacity ${ }^{13-15}$. Thus, DCs may be central to both onset and progression of RA and could be a logical target for treatment.

Tonicity-responsive enhancer-binding protein (TonEBP), also known as nuclear factor of activated T-cells 5 (NFAT5), belongs to the Rel family of transcriptional factors, which includes nuclear factor-kB (NF-kB) and NFAT1-4 ${ }^{16,17}$. TonEBP was identified initially as the central regulator of cellular responses to hypertonic stress ${ }^{16,18,19}$. Recent emerging studies show the role of TonEBP in the development and activation of immune cells, particularly T-cells and macrophages in osmostress-dependent and osmostressindependent contexts ${ }^{20,21}$. TonEBP induces activation of pathogenic Th17 cells ${ }^{22,23}$ and pro-inflammatory macrophages $^{24-26}$. As a result, numerous studies in humans and mice show that increased expression of TonEBP contributes to inflammatory and autoimmune diseases ${ }^{26-30}$. Conversely, downregulation of TonEBP reduces inflammation, thereby helping to prevent these diseases ${ }^{27-30}$. Emerging data indicate that single-nucleotide polymorphisms in TONEBP are associated with inflammation ${ }^{28}$, diabetic nephropathy ${ }^{28,31}$ and risk of type 2 diabetes mellitus ${ }^{32}$ in various human cohorts suggesting that variations in the level of TonEBP expression affect disease susceptibility ${ }^{33}$.

TonEBP is highly expressed in macrophages obtained from the synovium of patients with RA than in normal macrophages from healthy individuals ${ }^{27}$. Global TonEBP haplo-insufficiency in a mouse model of RA markedly prevented pannus formation and cartilage destruction, which was related to the reduced survival and proinflammatory activation of macrophages ${ }^{27,30}$. While the role of TonEBP in macrophages is well-established, its role in DCs is unclear. Here, we examined the intrinsic role of TonEBP in the maturation and functioning of DCs in the context of inflammatory arthritis. Lack of TonEBP in myeloid cells, including DCs and macrophages, alleviated disease severity in mouse models of inflammatory arthritis, as well as inhibited maturation of DCs and differentiation of Th1 and Th17 cells in draining LNs and inflamed joints. Importantly, we found that TonEBP promotes maturation and inflammatory responses of DCs in response to toll-like receptor 4 (TLR4) stimulation, and then it induces differentiation of pro-inflammatory Th1 and Th17 cells via p38 mitogen-activated protein kinase (MAPK).

\section{Results}

TonEBP-deficient myeloid cells reduce the severity of arthritis in mouse models

The blockade of RA development in TonEBPhaplodeficient mice ${ }^{27,30}$ led us to examine the role of myeloid TonEBP in a mouse model of inflammatory arthritis based on myeloid-specific TonEBP knockout; these mice are referred to as TonEBP ${ }^{f l f l}$ LysM-cre mice. First, we generated TonEBP $P^{f l f l} L y s M$-cre mice using the Cre-lox system (TonEBP ${ }^{f l / f}$; lysozyme 2 promoter drivenCre). Floxed TonEBP mice that did not express Cre recombinase (TonEBP $P^{f l f}$ alone) were used as a control. In myeloid lineage cells (peritoneal macrophages, and bone marrow-derived macrophages (BMDMs) and bone marrow-derived-dendritic cells (BMDCs)) TonEBP levels were dramatically reduced in the TonEBP ${ }^{f / f l}$ LysM-cre mice compared to their TonEBP $P^{f l / f l}$ littermates (Supplementary Fig. 1a) confirming genetic deletion of TONEBP.

We examined whether TonEBP expressed by myeloid cells plays a role in the collagen-induced arthritis (CIA) model, which is associated with autoimmunity to type II collagen. Mice were immunized with collagen, with a booster immunization given 14 days later (Supplementary Fig. 1b). Clinical signs of arthritis began to develop on Day 12 after the booster immunization and progressed to Day 28, the end of the experimental period (Fig. 1a, b). Disease severity in TonEBP $P^{f / f l}$ LysM-cre mice was lower than that in control mice at Day 16 after boosting; this difference persisted up to Day 28, although arthritis onset was comparable in both groups of mice up to Day 12 (Fig. 1a, b). These clinical assessments were supported by histological examination of representative ankle joints. On Day 28, control ankle sections showed clear evidence of bone destruction, inflammatory cell infiltration, and synovial hyperplasia, all of which were markedly less severe in TonEBP $P^{L y s M-K O}$ mice (Fig. 1c). Less cartilage damage was also observed in TonEBP ${ }^{f l f l}$ LysM-cre mice (Fig. 1d). Next, we measured serum levels of anti-collagen II (CII) antibodies and inflammatory mediators (IL- $1 \beta$, TNF- $\alpha$, and MCP-1), which play an important role in the pathogenesis of $\mathrm{CIA}^{10}$. CIIspecific IgG1 and IgG2c levels in TonEBP $P^{f l / f l} L y s M$-cre mice were markedly lower than those in control mice with CIA (Fig. 1e). Serum levels of IL-1 $\beta$, TNF- $\alpha$, and MCP-1 were also lower in TonEBP $P^{f l / f l} L y s M$-cre mice (Fig. 1f). We also examined the role of TonEBP in an adjuvant-induced arthritis (AIA) model. TonEBP $P^{f l / f l}$ LysM-cre mice and littermate control mice immunized with complete Freund's adjuvant (CFA) development arthritis; progression was monitored by measuring paw volume for 14 days (Supplementary Fig. 1c). We noted a marked increase in the paw volume of control mice from 3 to 14 days post-CFA injection; however, the increase in hind paw volume of $T o n E B P^{f l / f l} L y s M$-cre mice was significantly lower than that in control mice (Supplementary Fig. 1d, e).

Taken together, these data demonstrate that the reduction of arthritis severity and inflammatory responses in TonEBP $P^{f l / f l} L y s M$-cre mice phenocopies those in global TonEBP-haplodeficient mice ${ }^{27,30}$, and that TonEBP in myeloid cells increases severity of arthritis. 


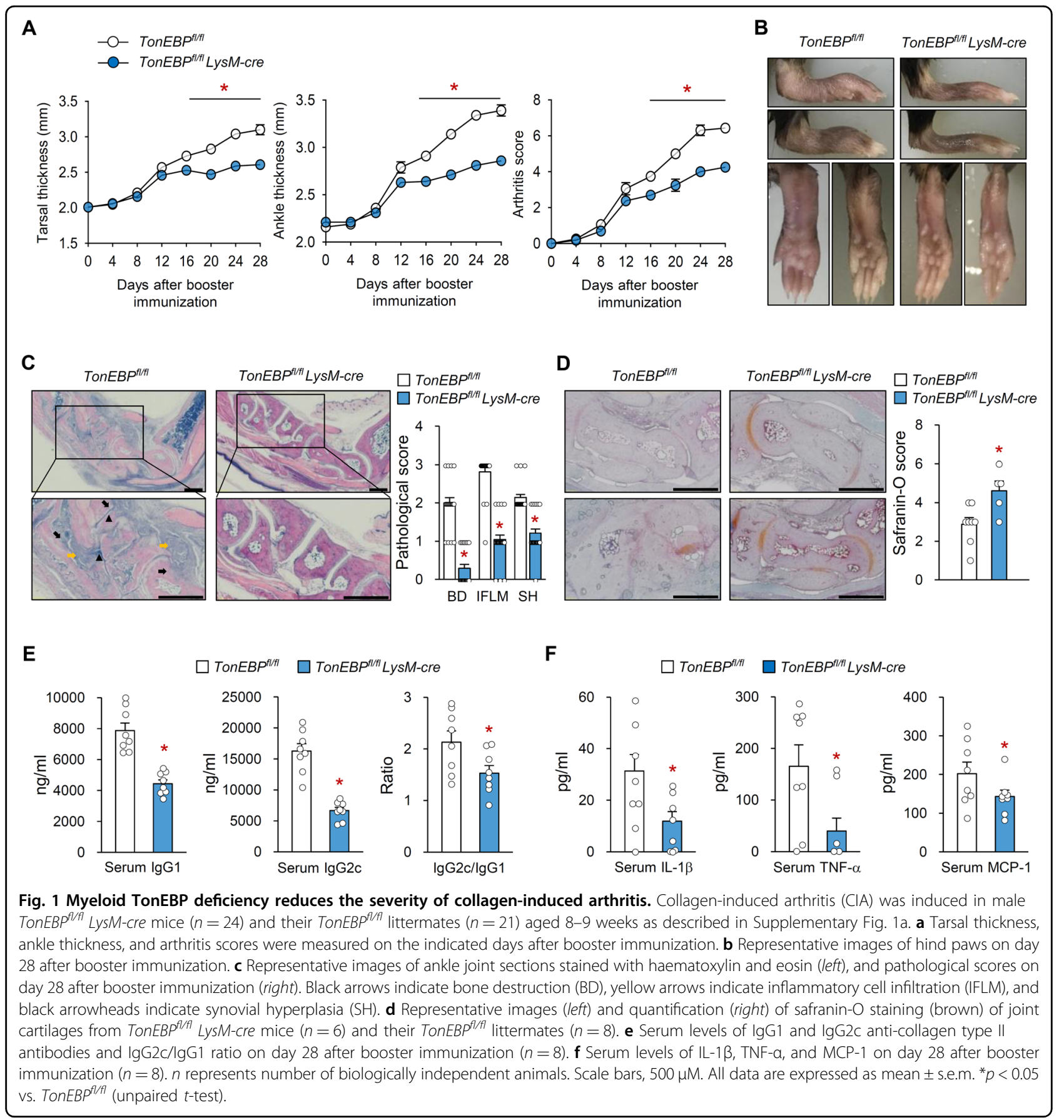

Deficiency of myeloid TonEBP inhibits immune responses in the paw tissue of CIA mice

Since TonEBP $P^{f l f l}$ LysM-cre mice showed less severe inflammation and bone destruction (Fig. 1), we next examined RA-related immune responses in paw tissue. As the CIA model mimics many features of human RA and involves both the innate and adaptive immune systems, we performed the following experiments using the CIA model. First, we analyzed expression of mRNA encoding TonEBP in paw extracts from normal and CIA mice. The levels of TonEBP mRNA in the paw tissue of normal mice were below the limit of detection (Ct-value $>40$ in $\mathrm{qPCR}$ ) and were higher in the paws of control mice with CIA (Supplementary Fig. 2). The levels in the paw tissue of TonEBP $P^{f l f l}$ LysM-cre mice were lower than those in the control mice (Fig. 2a).

Next, we examined expression of mRNA encoding IL$1 \beta$, IL-12, IL-18, IL-23, TGF- $\beta$, and MCP-1, all of which are inflammatory cytokines relevant to RA pathogenesis, in paw tissue from mice with CIA. The paw tissue of 


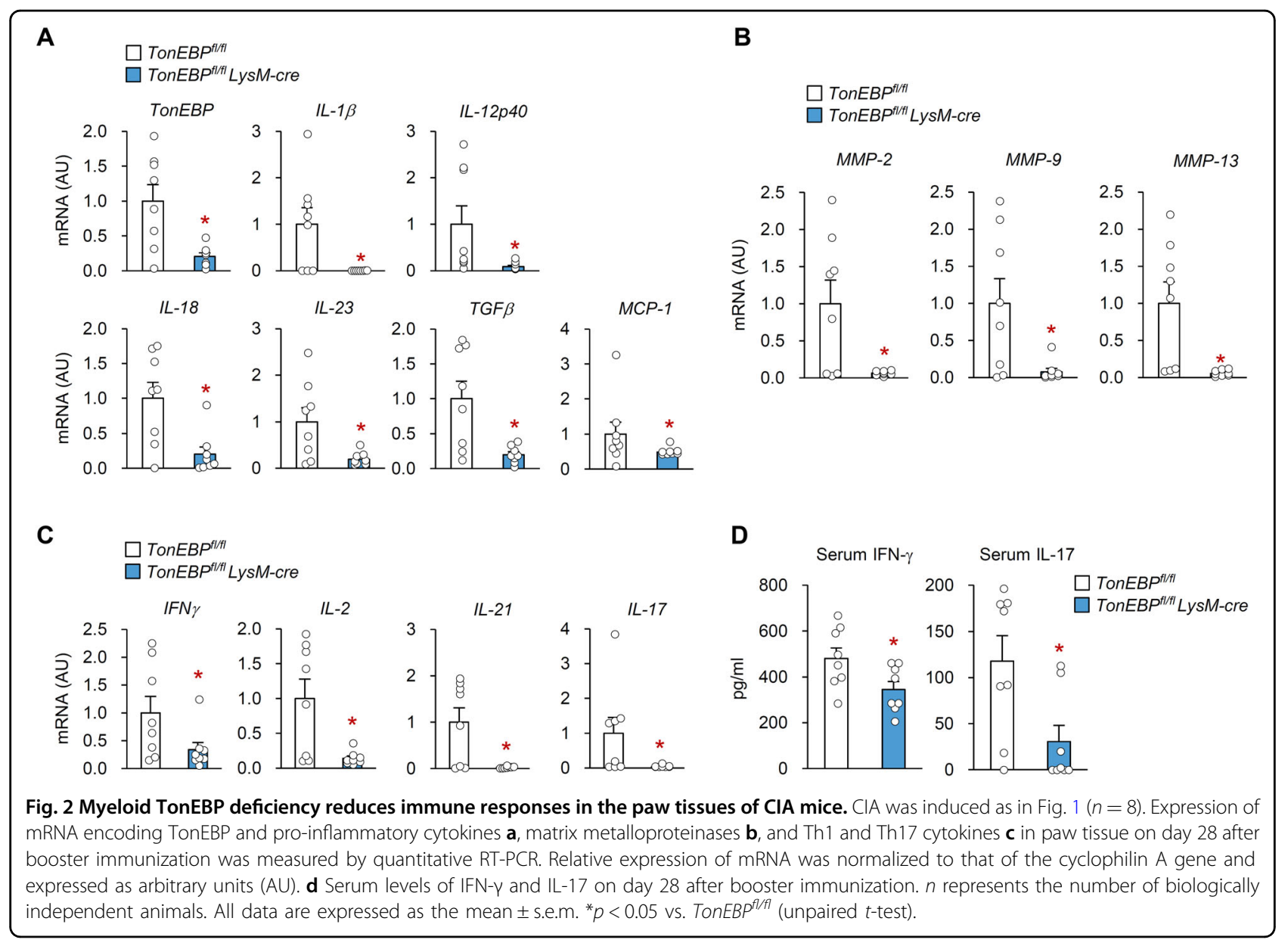

normal mice exhibited undetectable levels of mRNA encoding any of these genes, whereas expression in CIA mice increased markedly (Supplementary Fig. 2a). Notably, expression of mRNA encoding these cytokines was markedly lower in TonEBP $P^{f l f l} L y s M$-cre mice than in littermate controls (Fig. 2a). Consistent with the observed cartilage destruction (Fig. 1d), mRNA encoding matrix degrading enzymes (matrix metalloproteinase (MMP)-2, MMP-9, and MMP-13) was abundant in mice with CIA (Supplementary Fig. 2b) but much lower in TonEBP $f^{f l / f l}$ LysM-cre mice (Fig. 2b).

Given the importance of Th1 and Th17 cell activation in the pathogenesis of RA human patients and CIA mice ${ }^{34,35}$, we examined expression of mRNA encoding Th1 (IFN- $\gamma$ and IL-2) and Th17 (IL-21 and Th17) cytokines, all of which have pro-inflammatory functions. These genes were highly expressed in the paw tissue of control CIA mice (Supplementary Fig. 2c) but much less so in the paw tissue of TonEBP $P^{f l / f l}$ LysM-cre mice (Fig. 2c). Consistent with these results, deletion of myeloid-specific TonEBP led to lower serum levels of IFN- $\gamma$ and IL-17 than in control mice with CIA (Fig. 2d). Collectively, these data demonstrate a key role for myeloid TonEBP in CIA- induced paw inflammation and associated immune responses.

\section{Myeloid deficiency of TonEBP inhibits maturation of DCs and T-cell activation in CIA mice}

Draining LN hypertrophy increased cellularity, and changes in the cellular composition of LNs are characteristics features of RA in humans and mice ${ }^{36,37}$. To assess whether TonEBP in myeloid cells affects hypertrophy of inguinal (i) LNs, we first examined the total cell number and weight of iLNs at Day 7 post-booster immunization with CII. iLNs from TonEBP $P^{f l f l} L y s M$-cre mice weighed less and contained fewer cells than iLNs from control mice with CIA (Fig. 3a). Because TonEBP $P^{f l / f l}$ LysM-cre mice produced less Th1 and Th17 cytokines (Fig. 2c and d), we next examined CII-specific CD4 ${ }^{+}$ $\mathrm{T}$-cell responses in iLNs. Flow cytometry analysis revealed lower numbers of Th1 and Th17 cells in iLNs from TonEBP $P^{f l f l}$ LysM-cre than those from control CIA mice (Fig. 3b and Supplementary Fig. 3a).

The maturation of DCs is the most important step in the trafficking of DC to the $\mathrm{LNs}^{38}$ and activation of naïve $\mathrm{T}$-cells ${ }^{39}$ during RA development. Therefore, we 


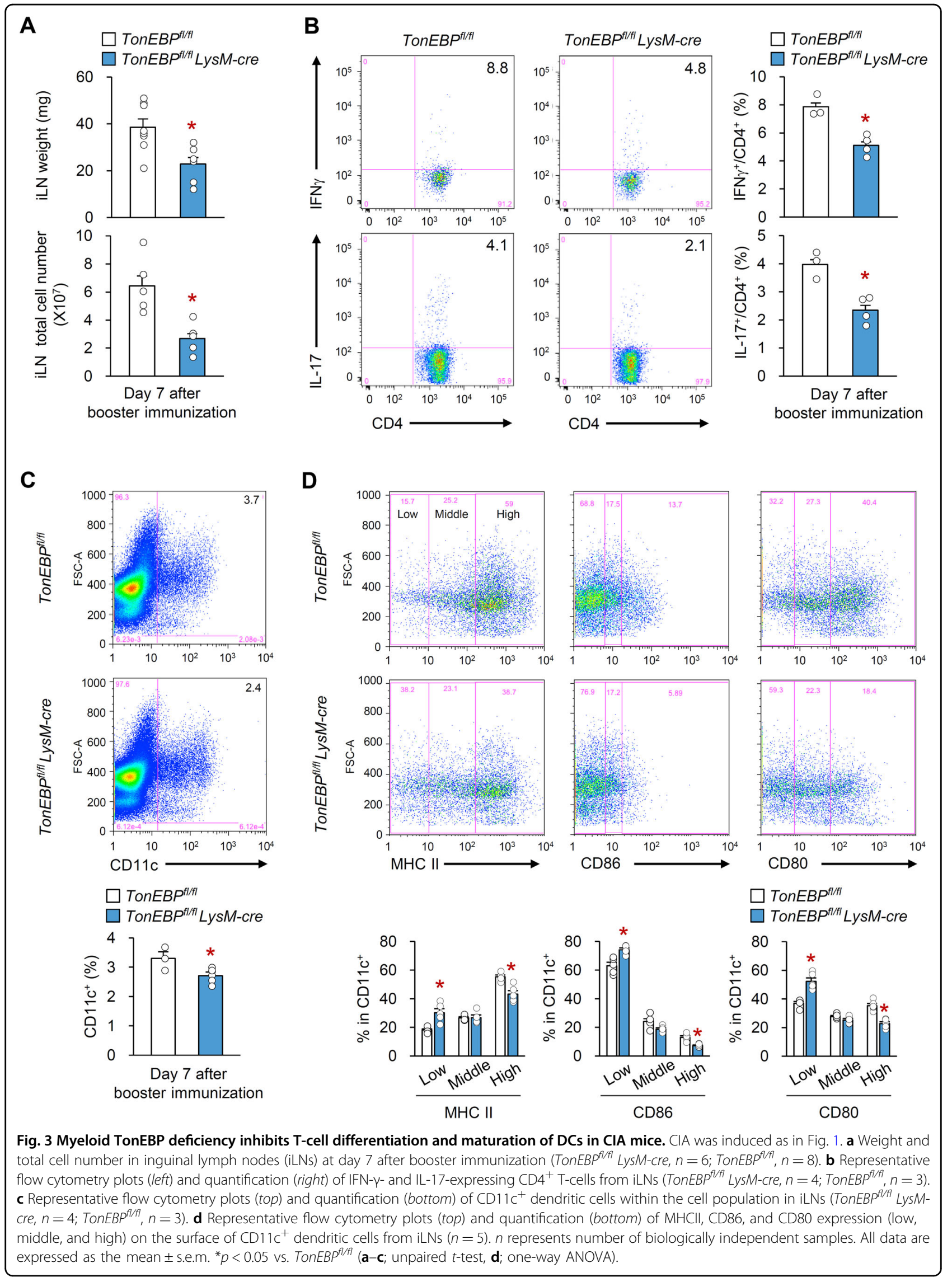


examined the effect of myeloid TonEBP depletion on the distribution of DCs in iLNs. iLNs from TonEBP $P^{f l / f l} L y s M$ cre mice contained fewer DCs than those from control CIA mice at Day 7 post-booster immunization (Fig. 3c and Supplementary Fig. 3b). Furthermore, DCs from the iLNs of TonEBP ${ }^{\text {flfl }}$ LysM-cre expressed lower levels of MHCII and co-stimulatory molecules (CD80 and CD86), which are the hallmark of DC maturation (Fig. 3d). Given the reduced numbers of DCs in iLNs from TonEBP $P^{f l / f l}$ LysM-cre mice (Fig. 3c), we also examined the gene expression of CC-chemokine receptor 7 (CCR7), which have an important role in trafficking of DC to LNs, and its ligands CC-chemokine ligand 19 (CCL19) and CCL2 $1^{40,41}$. There were no differences in the level of mRNA expression of these genes (Supplementary Fig. 3c) demonstrating that the myeloid TonEBP does not modulate the expression of CCR7, CCL19, and CCL21 in iLNs of mice with CIA.

Collectively these data suggest that myeloid TonEBP promotes maturation of DCs and activates T-cells in draining LNs of mice with CIA.

\section{TonEBP promotes DC maturation and inflammatory responses}

We next addressed the intrinsic role of TonEBP during DC maturation. To do this, we generated BMDCs by culturing BM from TonEBP $P^{f / f l} L y s M$-cre mice and their TonEBP $P^{f / f l}$ littermates with granulocyte/macrophage colony-stimulating factor (GM-CSF). On day 6 of culture with GM-CSF, non-adherent and loosely adherent cells were transferred to Petri dishes to remove $\mathrm{BMDMs}^{42,43}$. After 1 day of culture, non-adherent cells and adherent cells were harvested separately. The non-adherent cells expressed higher levels of CD11c, MHCII, and costimulatory molecules (CD86 and CD80) but lower levels of F4/80 compared to adherent cells (Supplementary Fig. 4a). Furthermore, the levels of DC markers RelB (a transcription factor of DC), CCR7, CD86, and CD80 were significantly higher in non-adherent cells than adherent cells (Supplementary Fig. 4b). In contrast, expression of macrophage markers MafA and MafB was lower in the non-adherent cells (Supplementary Fig. 4c). Thus, the non-adherent cells were highly enriched for DCs.

We first examined the effect of TonEBP on development of DC from BM. Notably, GM-CSF cultures of BM of TonEBP ${ }^{f l / f l}$ LysM-cre and control mice had similar number of BMDC and comparable percentage of CD11c expression (Supplementary Fig. 4d). Furthermore, TonEBP-deficient CD11c ${ }^{+}$BMDCs displayed similar levels of MHCII, CD86, and CD80 under steady-state conditions (Fig. 4a). Similarly, expression of mRNA encoding MHCII-related genes ( $\mathrm{H} 2-\mathrm{Aa}, \mathrm{H} 2-\mathrm{Ab}$, and CD74) in BMDCs was not affected by lack of TonEBP; however, TonEBP-deficient BMDMs showed reduced expression of mRNA encoding CD74, $\mathrm{H} 2-\mathrm{Aa}$, and $\mathrm{H} 2-\mathrm{Ab}$, a finding in line with a previous report (Supplementary Fig. $4 \mathrm{e})^{44}$. These data suggest that lack of TonEBP does not affect DC development and homeostasis.

Next, to address whether TonEBP is involved in DC maturation and inflammatory responses, we stimulated BMDCs with the TLR4 ligand, lipopolysaccharide (LPS), which is a potent inducer of DC maturation and inflammatory responses ${ }^{45}$. Upon stimulation with LPS for $24 \mathrm{~h}$, the percentage of cells expressing high levels of MHCII, CD86, and CD80 protein increased, whereas that of cells expressing low and intermediate levels decreased (Supplementary Fig. 5a). Notably, fewer BMDCs from TonEBP $P^{f l / f l} L y s M$-cre mice expressed high levels of these proteins relative to BMDCs from control mice, whereas the percentage of cells expressing low levels of these proteins was higher than that in control mice (Fig. $4 \mathrm{~b}$ ). The lack of TonEBP expression in BMDCs did not affect expression of gene encoding TLR4 and CD14, a coreceptor for TLR4 activation (Supplementary Fig. 5b). In addition, cell viability and apoptosis in BMDCs from TonEBP $P^{f l f l}$ LysM-cre mice were comparable to those in control mice under basal and LPS-stimulated conditions (Supplementary Fig. 5c, d). These data suggest that TonEBP plays a critical role in the maturation of BMDC in response to LPS.

We also examined expression of pro-inflammatory cytokines by LPS-stimulated BMDCs. Expression of TonEBP mRNA and protein increased after LPS stimulation; however, a significant reduction was observed in TonEBP-deficient BMDCs (Fig. 4c). As expected, LPStreated control BMDCs expressed high levels of proinflammatory cytokines IL-1 $\beta$, IL-12, IL-6, and IL-23; however, levels were lower in TonEBP-deficient BMDCs (Fig. 4c). On the other hand, expression of mRNA encoding CCR7 was not affected by TonEBP deficiency (Fig. 4c) in line with results from iLNs (Supplementary Fig. 3c).

Collectively, these data demonstrate that TonEBP promotes maturation and inflammatory responses by BMDCs in response to LPS.

TonEBP in DCs promotes proliferation of $\mathrm{CD}^{+} \mathrm{T}$-cells and subsequent differentiation into Th1/Th17 cells

Next, we asked whether TonEBP in DCs is responsible for differentiation of Th1 and Th17 cells. We isolated $\mathrm{CD}^{+}{ }^{+} \mathrm{T}$-cells from CII-immunized wild-type mice and co-cultured them with LPS plus CII-stimulated BMDCs isolated from TonEBP ${ }^{f l / f l}$ or TonEBP $P^{f l / f l} L y s M$-cre mice for 4 days. We examined proliferation of $\mathrm{CD}_{4}^{+} \mathrm{T}$-cells and found that cells co-cultured with TonEBP-deficient BMDCs proliferated markedly less well than those from control BMDC at 2-4 days (Fig. 5a). In addition, the percentages of $\mathrm{IFN \gamma}^{+}$and $\mathrm{IL}^{-} 7^{+}$cells within the 


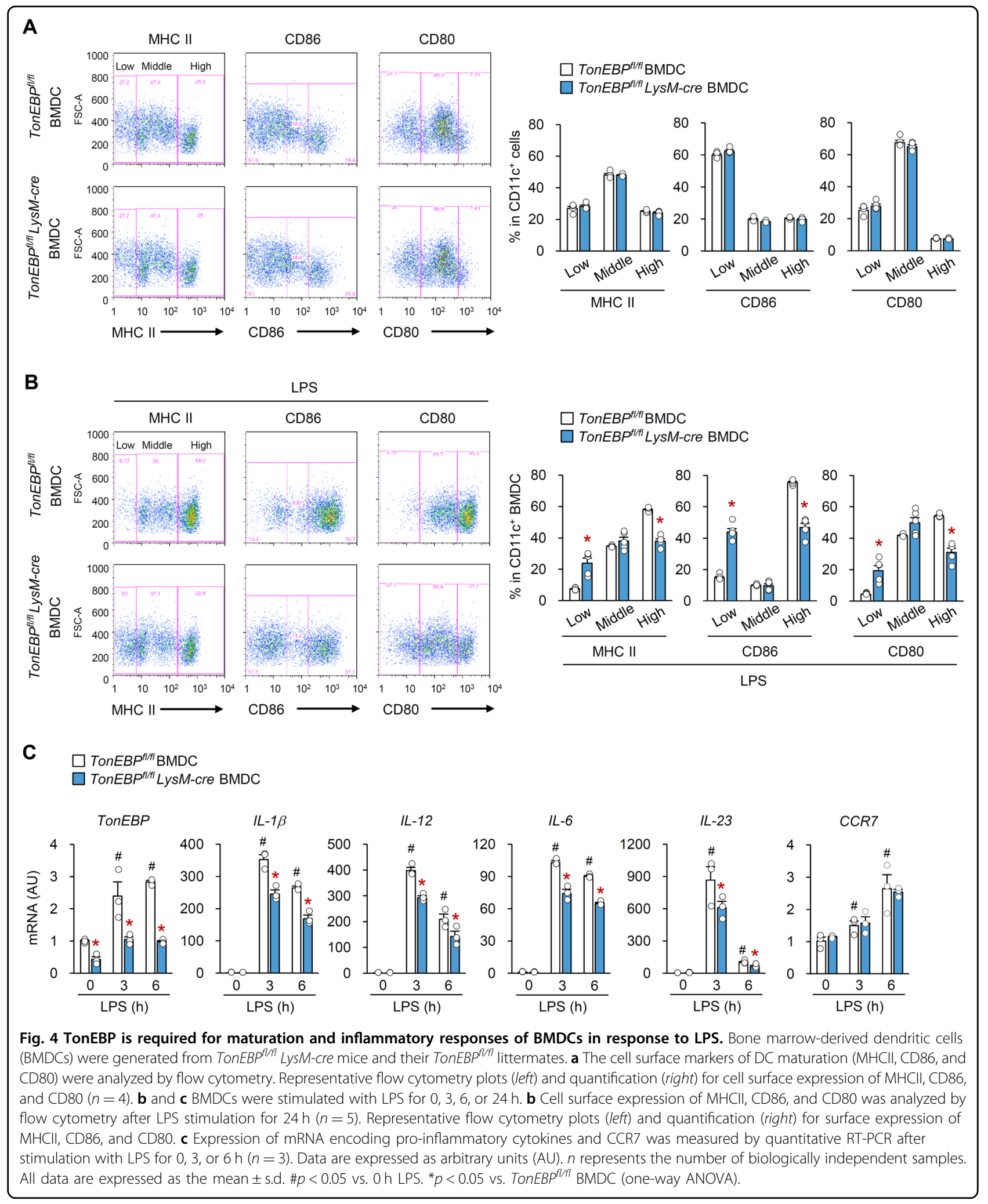

$\mathrm{CD}^{+}$T-cells population co-cultured with TonEBPdeficient BMDCs were reduced (Fig. 5b). Consistent with this, $\mathrm{CD} 4^{+}$T-cells co-cultured with TonEBP-deficient
BMDCs secreted less IFN $\gamma$ and IL-17 (Fig. 5c). These data suggest that TonEBP in DCs is required to induce $\mathrm{CD} 4^{+}$ $\mathrm{T}$-cell proliferation and differentiation into Th1/Th17 cells. 
A

$-\square$ CD4 $^{+} T$ cells only
$-\square \mathrm{CD}^{+} \mathrm{T}$ cells co-cultured with TonEBP ${ }^{t / f i} \mathrm{BMDC}$

$-\square \mathrm{CD4}^{+} \mathrm{T}$ cells co-cultured with TonEBP ${ }^{\text {fl/fi }}$ LysM-cre BMDC
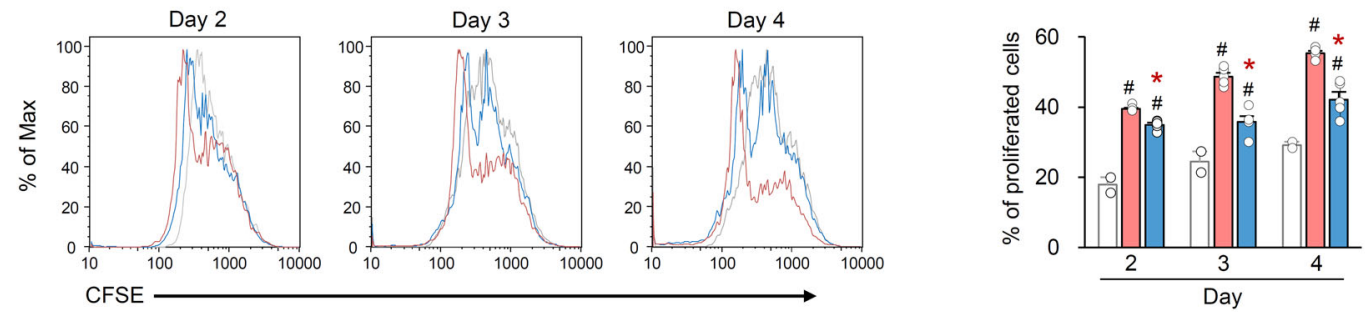

B
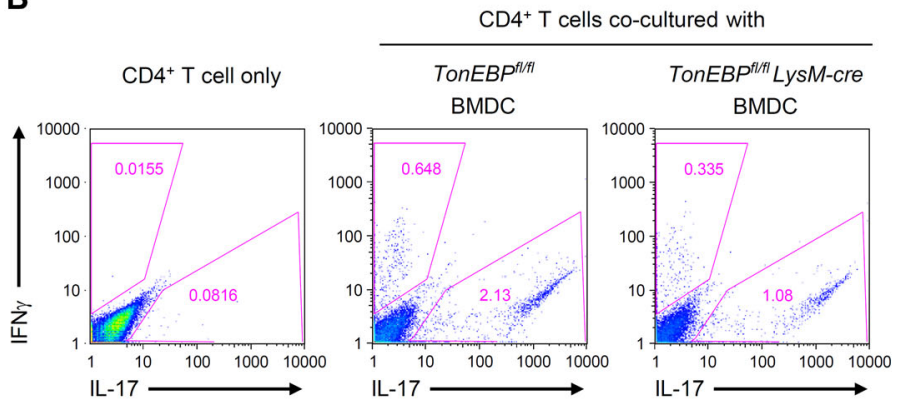

C

$\square \mathrm{CD}^{+} \mathrm{T}$ cells + TonEBP $P^{f / i l} \mathrm{BMDC}$

$\mathrm{CD}^{+} \mathrm{T}$ cells + TonEBP ${ }^{f / f i l}$ LysM-cre BMDC
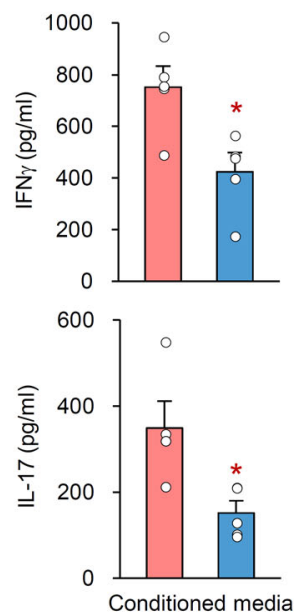

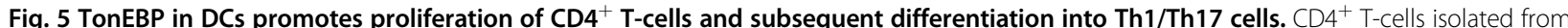
Cll-immunized wild-type mice were co-cultured with LPS/CII-stimulated BMDCs isolated from TonEBP $P^{f / / f l}$ LysM-cre mice and TonEBP $P^{f / f}$ littermates. $\mathrm{CD}^{+}$T-cells alone without BMDCs were used as a control. a Proliferation of CD4 ${ }^{+}$T-cells was assessed based on reduction of fluorescence of 5(6)carboxyfluorescein diacetate succinimidyl ester (CFSE) at 2, 3, or 4 days $(n=5)$. Representative flow cytometry plots (left) and quantification (right) of the percentage of proliferated CD4 ${ }^{+}$T-cells. b Representative flow cytometry plots (top) and quantification (bottom) of the percentage of IFN ${ }^{+}$and $\mathrm{IL}-17^{+}$cells within the CD4 ${ }^{+}$T-cell population $(n=4)$. c Concentrations of IFNy and IL-17 in conditioned medium from CD4 ${ }^{+}$T-cells co-cultured with LPS/Cll-stimulated BMDCs $(n=5)$. $n$ represents the number of biologically independent samples. All data are expressed as the mean $\pm s . d$. \#p $<0.05$ vs. $C D 4^{+}$T-cells only. ${ }^{*} p<0.05$ vs. TonEBP ${ }^{f / f l}$ BMDC (unpaired t-test).

TonEBP in DCs regulates LPS-mediated DC maturation via p38 MAPK

Next, we examined potential pathways that mediate TonEBP function during maturation of BMDCs in response to LPS. Because lack of TonEBP expression by BMDCs attenuated surface expression of maturationrelated molecules in response to LPS (Fig. 4b), we first examined expression of genes encoding MHC II. TonEBP-deficient DCs did not show altered expression of genes encoding MHC II proteins or its transcriptional coactivator class II transactivator (CIITA) regardless of LPS treatment (Supplementary Fig. 6a); by contrast, their expression in TonEBP-deficient BMDMs decreased (Supplementary Fig. 6b), as previously reported ${ }^{42}$. This indicates that TonEBP affects cell surface expression of
DC maturation markers in response to LPS without changes in the level of expression. Because the surface expression is dependent on MAPKs ${ }^{46,47}$, we next assessed whether TonEBP modulates activation of MAPKs (ERKs, JNKs, and p38) in DCs stimulated by LPS. As expected, LPS activated all MAPKs in control BMDCs (Fig. 6a). Interestingly, phosphorylation of p38 (but not ERKs or JNKs) in TonEBP-deficient cells was lower than that in control BMDCs (Fig. 6a), suggesting that TonEBP plays a role in p38 MAPK activation.

To test whether TonEBP-mediated DC maturation is dependent on the p38 MAPK pathway, we pretreated control and TonEBP-deficient BMDCs with SB203580, an inhibitor of p38 MAPK, and then stimulated them with LPS. Surface expression of MHCII, CD86, and CD80 in response to LPS 


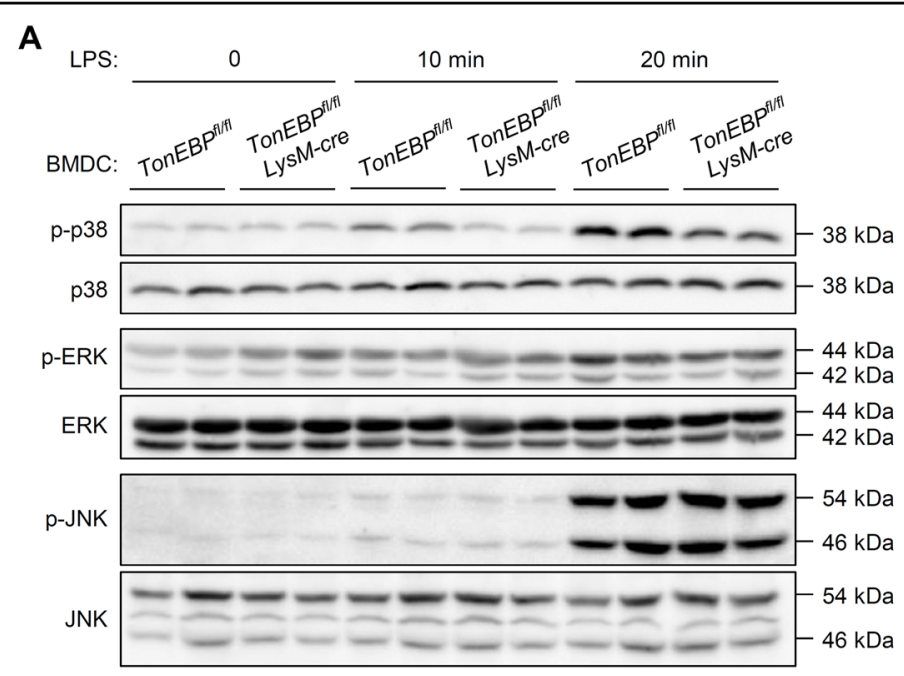

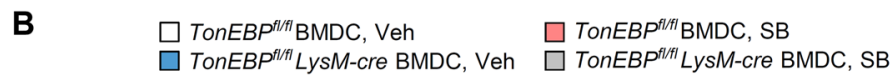
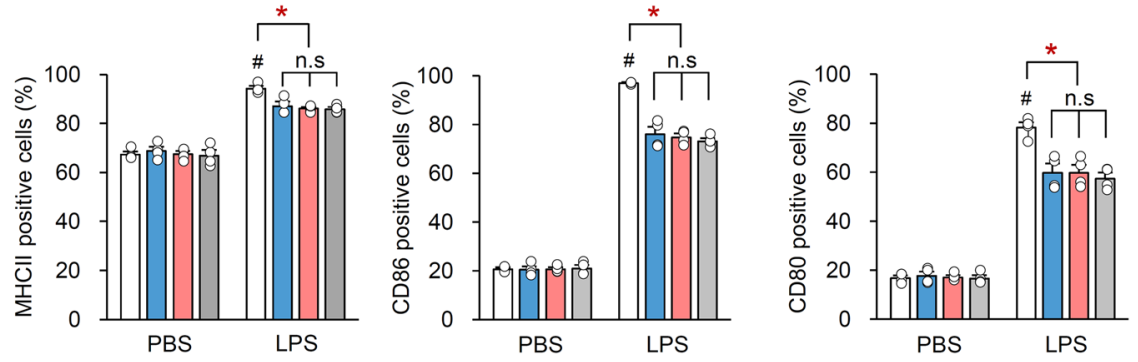

C

$\square$ T cells + TonEBP $P^{\text {fl/fl}}$ BMDC $\square$ T cells + TonEBP fl/fI $L y s M-c r e ~ B M D C$
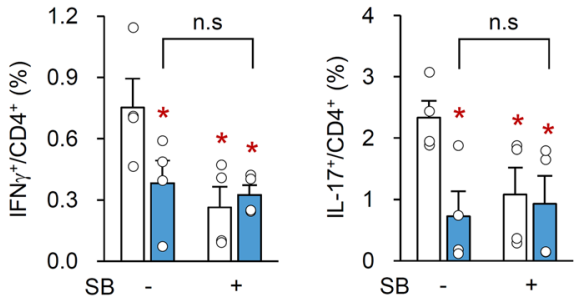

D

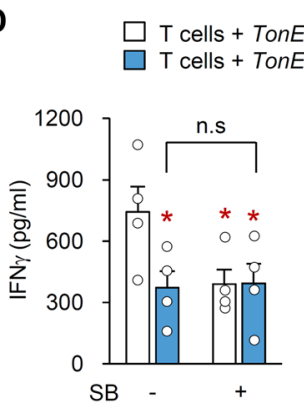
$E B P^{f / / f I}$ LysM-cre BMDC

Fig. 6 TonEBP in DCs controls LPS-mediated DC maturation via p38 MAPK. BMDCs were generated from TonEBP ${ }^{f / f l}$ Lys littermates followed by stimulation with LPS for 0, 10, 20 min, or $24 \mathrm{~h}$. a Representative immunoblots showing activation of p38, ERK, and JNK MAPKs after 0 , 10, or 20 min of LPS-stimulation. b BMDCs were pretreated with SB203580 (SB), an inhibitor of p38 MAPK, followed by LPS stimulation for 24 h. Cell surface expression MHCII, CD86, and CD80 was analyzed by flow cytometry. c and $\mathbf{d}$ BMDCs were incubated with LPS/CII or LPS/CII/SB for $24 \mathrm{~h}$ followed by co-culture with CD4 ${ }^{+}$T cells for 3 days. $\mathbf{c}$ The percentages of IFNY ${ }^{+}$and IL-17 ${ }^{+}$cells within the CD4 ${ }^{+} \mathrm{T}$ cell population $(n=4)$. $\mathbf{d}$ Levels of IFNy and IL-17 protein in culture medium from CD4 ${ }^{+}$T cells co-cultured with BMDCs $(n=4)$. $n$ represents the number of biologically independent

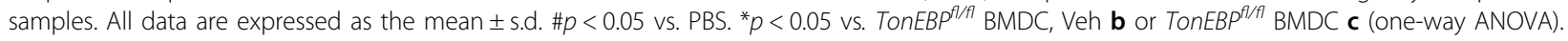

was reduced markedly by TonEBP deficiency or addition of SB203580 (Fig. 6b). Notably, inhibition of p38 MAPK had no additional effect on reduced surface expression of MHCII, CD86, and CD80 induced by TonEBP deficiency (Fig. 6b). Neither TonEBP deficiency nor SB203580 affected the surface expression of MHCII, CD86, and CD80 by cells treated with PBS (Fig. 6b). Furthermore, to examine the effect of
SB203580 on TonEBP-mediated differentiation of $\mathrm{CD}^{+}$ $\mathrm{T}$-cells, we incubated BMDCs from TonEBP fl/fl or TonEBP ${ }^{f l / f l}$ LysM-cre mice for $24 \mathrm{~h}$ with LPS plus CII, or with LPS, CII, and SB203580, and then co-cultured them with CD4 ${ }^{+}$T-cells for 3 days. SB203580 had no additional effect on differentiation into Th1 and Th17 cells (Fig. 6c) or on secretion of IFN- $\gamma$ and IL-17 (Fig. 6d) over those 


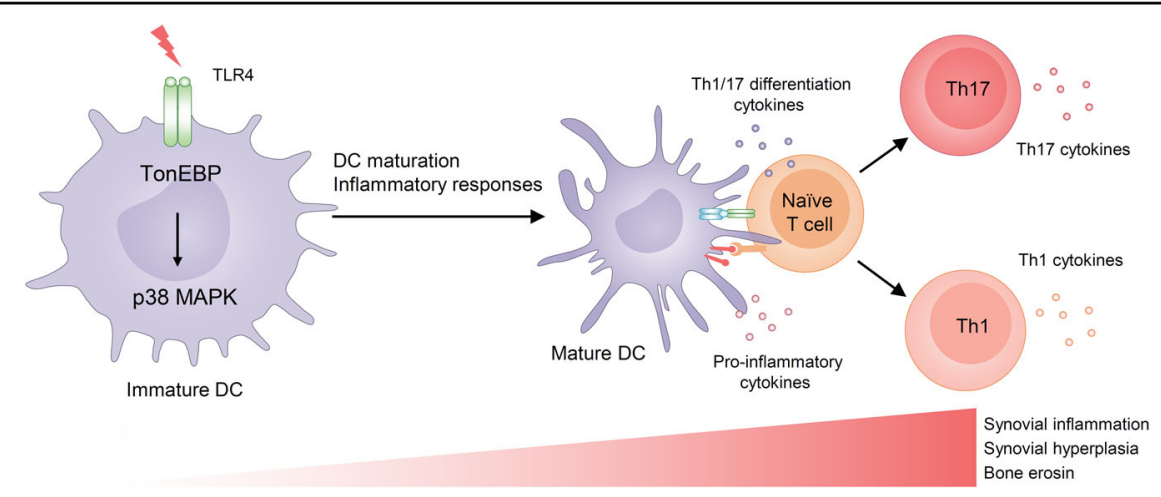

Fig. 7 TonEBP expression by DCs promotes maturation and contributes to development of rheumatoid arthritis. TonEBP expression by dendritic cells (DCs) promotes maturation and inflammatory responses in response to TLR4 stimulation via activation of p38 MAPK. Maturation of DCs stimulates differentiation of $\mathrm{CD}^{+}$naïve T-cells into Th1 and Th17 cells, leading to the development of rheumatoid arthritis.

observed in $\mathrm{CD}^{+}$T-cells co-cultured with TonEBPdeficient BMDCs. Collectively, these data demonstrate that TonEBP promotes activation of DCs in response to LPS by activating p38 MAPK.

\section{Discussion}

Previously, we reported that global TonEBP haploinsufficiency protected mice against RA, and that this was related to reduced survival and activation of macrophages $^{27,30}$. Here, we used myeloid-specific TonEBPdeficient mice to show that TonEBP expressed by myeloid cells, including macrophages and DCs, contributes to progression of RA. Moreover, we reveal the intrinsic functions of TonEBP during DC maturation and inflammatory responses. TonEBP expressed by DCs promotes TLR4-mediated maturation via p38 MAPK and, as a result, increases pro-inflammatory $\mathrm{CD} 4 \mathrm{~T}$-cell responses (Fig. 7).

A role for TonEBP in DCs is suggested by the crucial role played by DCs during pathogenesis of most autoimmune diseases, including $\mathrm{RA}^{3}$. Autoimmune diseases are caused by disruption of immune tolerance to selfantigens, resulting in damage to (or dysfunction of) normal tissues. The dysregulated activity of DCs breaks selftolerance; thus DCs are an attractive therapeutic target. Indeed, some treatments for autoimmune diseases target DCs. TNF- $\alpha$ blockade, an effective therapy for RA, acts by impairing the survival and function of DCs ${ }^{48}$. Therapeutic vaccination with IL-27-conditioned DCs suppresses the development of experimental autoimmune encephalomyelitis by reducing pathogenic $\mathrm{T}$-cell responses ${ }^{49}$. IFN- $\beta$, a first-line disease-modifying therapy for multiple sclerosis, reduces migration of DCs to the draining LNs and reduced their capacity to activate $\mathrm{CD} 4^{+}$effector $\mathrm{T}$-cells $\mathrm{s}^{50}$. Therefore, identification of TonEBP as a novel regulator of DC maturation and function provides new insight into the mechanisms underlying the development of autoimmune diseases, and may guide possible therapeutic approaches. More importantly, our finding that DCs lacking TonEBP express normal levels of MHC class II and co-stimulatory molecules (Fig. 4a) is of great interest because DCs also play a key role in maintaining immune tolerance under steady-state conditions, i.e., in the absence of overt infection or inflammation ${ }^{51}$. Thus, this study provides an opportunity to further our understanding of the role of DC-intrinsic TonEBP in immune tolerance and autoimmunity.

Based on several lines of evidence from the present and previous works ${ }^{27,28,30}$, we identified TonEBP-dependent cellular immune responses that are potential new targets for therapeutic intervention in RA. First, genetic variants of TonEBP in a cohort of healthy humans are associated with expression of systemic inflammatory factors, including serum MMP and IL- $1 \beta^{28}$. Importantly, these genes increase the risk of RA in humans ${ }^{4}$. Our present and previous studies show that TonEBP deficiency reduces the severity of arthritis in mouse models due to reduced expression of inflammatory factors ${ }^{27,30}$, suggesting that genetic variability in expression of TonEBP leads to differential susceptibility for RA. Second, TonEBPdeficient DCs (in this study) and macrophages ${ }^{44}$ attenuate activation of pro-inflammatory effector T-cells, the causative agents of RA. Third, many TonEBP-dependent genes in DCs and macrophages are linked functionally to human arthritis or experimental arthritis. Taken together, these data suggest that targeting TonEBP may be of benefit to individuals with arthritis.

DCs and macrophages play important roles in the pathogenesis of $\mathrm{RA}^{52,53}$. DCs and macrophages arise from a common progenitor and share a variety of roles, including antigen presentation, participation in $\mathrm{T}$-cell homeostasis, and maintenance of immunity, while at the same time maintaining distinct functions within the immune system and in the context of disease ${ }^{3,54,55}$. MHC 
class II molecules are constitutively expressed on the cell surface of DCs and macrophages, and expression increases in response to cell-specific stimuli. Thus, these cells present antigens to $\mathrm{CD} 4^{+} \mathrm{T}$-cells ${ }^{3,56}$. Interestingly, the present and previous findings demonstrate that DCs and macrophages differ with respect to their dependence on TonEBP for MHC class II expression. Here, we found that TonEBP deficiency did not affect constitutive expression of MHC class II molecules by DCs under basal conditions (Fig. 4a); however, it did inhibit LPS-inducible expression of MHC II (Fig. 4b). By contrast, we and others ${ }^{44}$ showed that macrophages lacking TonEBP show reduced basal and IFN- $\gamma$-induced expression of mRNA encoding MHC class II, as well as surface expression of the protein. Furthermore, TonEBP expression by DCs promotes cell surface expression of MHC class II and co-stimulatory molecules in response to LPS via p38 MAPK. This finding is interesting because TonEBP in macrophages did not affect activation of p38 MAPK in response to LPS $^{25}$. These data suggest that TonEBP constitutes a cell-specific mechanism for pro-inflammatory activation of DCs.

In summary, our results reveal that the essential nature of TonEBP expression by DCs is to induce differentiation of effector T-cells. These findings have therapeutic implications in that targeting TonEBP in DCs may be an approach to treating disorders of the immune system and immune-associated diseases.

\section{Materials and methods \\ Mice}

All studies used male C57BL/6J mice. Mice carrying the loxP-targeted TonEBP gene (TonEBP $P^{f l f l}$ ) have been described $^{57}$ and were provided by Dr. Neuhofer. Lysozyme 2-cre knock-in mice, known as LysM-cre mice, were obtained from Jackson Laboratories (Bar Harbor, ME, USA). TonEBP ${ }^{f l f l}$ mice were crossed to LysM-cre mice to generate mice lacking TonEBP in myeloid cells. Agematched and sex-matched littermate control animals were used for all experiments. Animals were randomly assigned to experimental groups based on body weight. Blinding was not used. All animal procedures were approved by and performed according to guidelines of the Institutional Animal Care and Use Committee of the Ulsan National Institute of Science and Technology (UNISTACUC-16-08).

\section{Collagen-induced arthritis}

Male TonEBP ${ }^{f l / f l}$ LysM-cre mice and littermate control TonEBP $P^{f l / f l}$ mice (aged 8-9 weeks) were immunized by intradermal injection (at the tail base) of $100 \mu \mathrm{g}$ chicken type II collagen (CII; Chondrex, Redmond, WA, USA) emulsified in CFA containing $100 \mu \mathrm{g}$ of heat-killed Mycobacterium tuberculosis H-37RA (Chondrex). Mice received a booster immunization of $\mathrm{CII}$ in incomplete
Freund's adjuvant (IFA; Chondrex) into the left hind paw on Day 14. Paw swelling was measured at the indicated times using a caliper. On Day 28 after booster immunization, arthritis scores were graded 0-4 (1) $0=$ normal; $1=$ mild inflammation of a single area (i.e., midfoot, ankle, or toes); $2=$ moderately severe arthritis involving toes and ankle or midfoot; $3=$ severe arthritis involving the entire paw; and $4=$ severe arthritis resulting in ankylosis and loss of joint movement. Scores were assigned by an investigator blinded to the mouse genotype. The hind paw in which the booster immunization had been administered was excluded from the evaluation. The right hind paw and forepaw were assessed; thus the maximum possible arthritis score was $8^{58}$. No statistical methods were used to pre-determine sample sizes, but the sample sizes are comparable to those in previous studies $^{27,30}$.

\section{Histology}

At Day 28 post-booster immunization, mice were sacrificed prior to histological analysis. Fore and hind limbs that received no booster immunization were fixed in $10 \%$ formalin, decalcified, dehydrated, and then embedded in paraffin. Paraffin sections were stained with hematoxylin and eosin (Sigma-Aldrich, Saint Louis, MO, USA) for morphological evaluation, or stained with safranin-O (Sigma-Aldrich) to examine glycosaminoglycan distribution in cartilage. Histological observation and pathologic scoring were performed by observation under a light microscope (X51; Olympus, Tokyo, Japan). The severity of three parameters of arthritis (bone erosion, joint infiltration by inflammatory cells, and synovial hyperplasia) was scored on a scale from 0 to 3 ( 0 , absent; 1 , weak; 2 , moderate; and 3 , severe $)^{59}$. For gene expression analysis (see below for details), paws were snap frozen in liquid nitrogen at the indicated times and subjected to mechanical disruption using a Polytron homogenizer (Kinematica AG, Luzern, Switzerland).

\section{Cell preparation and culture}

Single-cell solutions were prepared from iLNs draining the paw by digestion with collagenase $(1 \mathrm{mg} / \mathrm{ml})$ and DNase I $(0.1 \mathrm{mg} / \mathrm{ml})^{60}$. The total number of cells was determined by microscopic observation in a hemocytometer after trypan blue-staining. For cytokine detection, cell suspensions derived from iLNs were plated in 96-well plates and then stimulated for $5 \mathrm{~h}$ with cell activation cocktail containing $50 \mathrm{ng} / \mathrm{ml}$ phorbol 12-myristate 13acetate (Sigma-Aldrich) and $1 \mu \mathrm{g} / \mathrm{ml}$ ionomycin (SigmaAldrich), followed by stimulation for $12 \mathrm{~h}$ with GolgiStop $^{\mathrm{TM}}$ (BD Biosciences, San Jose, CA, USA). DCs and macrophages were generated from murine BM. Briefly, $\mathrm{BM}$ was flushed from the femurs of TonEBP $P^{f / f l}$ LysM-cre mice and littermate control TonEBP $P^{f l / f l}$ mice. A single-cell 
suspension was obtained by passing the BM through a 25 gauge needle and filtering through a cell strainer $(70 \mu \mathrm{m})$. The preparation was then divided into two separate cultures. BMDCs were generated as described $^{43,61}$ with slight modifications. BM were cultured in RPMI 1640 medium (Lonza, Cologne, Germany) supplemented with $10 \%$ heat-inactivated fetal bovine serum (FBS; ThermoFisher Scientific Inc., Waltham, MA, USA), penicillin/streptomycin $(100 \mathrm{U} / \mathrm{ml}$ and $100 \mu \mathrm{g} / \mathrm{ml}$, respectively; GE Healthcare, Salt Lake City, UT, USA), $55 \mu \mathrm{M} \beta$-mercaptoethanol (Life Technologies, Carlsbad, CA, USA), and $20 \mathrm{ng} / \mathrm{ml}$ mouse GM-CSF (PeproTech, Rocky Hill, NJ, USA) in tissue-culture-treated plates. On Days 2 and 4 of culture, floating cells were removed gently, and fresh medium was added. On day 6 of culture with GM-CSF, non-adherent and loosely adherent cells were cultured in Petri dishes for $24 \mathrm{~h}$ to allow GMBMDMs to adhere. Non-adherent cells were collected and used as BMDCs.

BMDMs were generated by culturing cells for 7 days in RPMI 1640 medium supplemented with 10\% heatinactivated FBS, penicillin/streptomycin, and 20\% L929 conditioned medium (as a source of macrophage colonystimulating factor) ${ }^{62}$. BMDCs and BMDMs were stimulated (or not) with LPS and then analyzed.

The experiments were repeated independently at least three times with at least three replicates. Each figure legend contained a sample size $(n)$ for each experimental condition, given as an exact number.

\section{DC and T-cell co-cultures}

Male C57BL/6 mice were immunized using an emulsion of chicken CII and CFA as described above. They then received a booster immunization of CII in IFA into the left hind paw on Day 8. Three days later, single-cell suspensions were prepared from iLNs by digestion with collagenase D. CD4 ${ }^{+}$ T-cells were obtained by positive selection on CD4Microbeads (Miltenyi Biotec, Bergisch, Germany). These cells were then labeled with $1 \mu \mathrm{M} 5(6)$-carboxyfluorescein diacetate succinimidyl ester (CFSE; Life Technologies, CA, USA). BMDCs from the TonEBP $P^{f l / f l} L y s M$-cre and littermate control TonEBP $P^{f l / f l}$ mice were cultured in 96-well plates $(1 \times$ $10^{5}$ per well) and stimulated with LPS $(100 \mathrm{ng} / \mathrm{ml})$ plus CII $(20 \mu \mathrm{g} / \mathrm{ml})$ for $24 \mathrm{~h}$. Next, cells were washed thoroughly and co-cultured with the CFSE-labeled $\mathrm{CD} 4^{+}$T-cells $\left(1 \times 10^{6}\right.$ per well) for 4 days. $\mathrm{CD} 4^{+} \mathrm{T}$-cells alone without BMDCs were used as a control. Cells were harvested at 2, 3, or 4 days and analyzed by flow cytometry.

\section{Phenotypic analysis of intracellular cytokine and surface marker expression}

Immunophenotyping of cells was performed by flow cytometry using multicolor fluorochrome-conjugated antibodies. Identification of cell surface markers was performed using antibodies purchased from BD Biosciences (San Jose, CA, USA; anti-CD11c FITC; clone HL3, anti-MHCII PE; clone 2G9, anti-CD86 APC; clone 2331 [FUN-1], anti-CD4 PerCP-Cy; clone RM4-5, anti-CD11b PerCP-Cy; clone M1/70, anti-F4/80 BV421; clone T452342) or BioLegend (San Diego, CA, USA; anti-CD80 PE/ Cy7; clone 16-10A1) and used at a dilution of 1:100-1:500 unless stated otherwise. After surface-staining, anti-IFN- $\gamma$ FITC (clone B27) and anti-IL-17 PE (clone TC11-18H10) (BD Biosciences) were used for intracellular staining using a fixation and permeabilization kit (BD Biosciences). Flow cytometry data were acquired on a BD LSR Fortessa (BD Biosciences), and data were analyzed using Flowjo software (Tree Star, Ashland, OR, USA).

\section{RNA isolation and reverse transcription PCR}

Total RNA was extracted from paw tissue, BMDCs, and BMDMs using TRIzol reagent (Invitrogen, Carlsbad, CA, USA), and complementary DNA was synthesized using M-MLV reverse transcriptase (Promega, Madison, WI, USA). Next, real-time PCR was performed using SYBR Green I Master and a LightCycler 480 II (Roche, Rotkreuz, Switzerland). The cycling conditions were as follows: $95^{\circ} \mathrm{C}$ for $5 \mathrm{~min}$, followed by 45 cycles of $95^{\circ} \mathrm{C}$ for $10 \mathrm{~s}$, $60^{\circ} \mathrm{C}$ for $15 \mathrm{~s}$, and $72{ }^{\circ} \mathrm{C}$ for $20 \mathrm{~s}$. Measured cycle threshold values were normalized to the cyclophilin $\mathrm{A}$ gene and expressed as fold changes relative to control samples. The primers are described in Supplementary Table 1.

\section{Immunoblot analysis}

Cells were washed twice with cold PBS and lysed in RIPA buffer (0.01 M Tris, pH 7.4, $0.15 \mathrm{M} \mathrm{NaCl}, 0.001 \mathrm{M}$ EDTA, 0.001 M EGTA, 1\% Triton-X 100; all from SigmaAldrich) containing 0.002 M PMSF (Sigma-Aldrich) and protease inhibitors (Roche). After centrifugation of the lysate, the supernatant was used for immunoblot analysis. The protein concentration was measured using the $\mathrm{BCA}$ protein assay system (Pierce Biotechnology, Rockford, IL, USA). Proteins were denatured in Laemmli buffer, and equal amounts from each sample were separated on SDS-polyacrylamide gels prior to transfer to PVDF membranes. Blocking, incubation with primary antibody, and washing of the membrane were performed in PBS supplemented with $0.05 \%$ Tween-20 (v/v) and 5\% (w/v) non-fat dry milk. The primary antibodies used for immunoblotting were anti-TonEBP (16; 1:3000), antiphospho-p38 (\#4511), anti-p38 (\#8690), anti-phospho-Erk (\#4370), anti-Erk (\#9102), anti-phospho-SAPK/JNK (\#4668), anti-SAPK/JNK (\#9252) (all from Cell Signaling Technology), anti-CREB (sc-377154, SantaCruz Biotechnology, Santa Cruz, CA, USA), and anti-phosphoCREB (sc-7978, SantaCruz Biotechnology). Anti-Hsc70 (\#200-301-A28; Rockland, Limerick, PA, USA) was used 
as a loading control. All antibodies were used at a 1:1000 dilution unless stated otherwise. Horseradish-peroxidaseconjugated goat anti-mouse (62-6520; ThermoFisher Scientific, Inc.) or anti-rabbit (65-6120; ThermoFisher Scientific, Inc.) secondary antibodies were diluted 1:5000. Reactive bands were detected by chemiluminescence using the ImageQuant LAS 4000 imaging system (GE healthcare).

\section{Cytokine analysis}

Cytokine (TNF- $\alpha$, IL-6, IL-17, IFN- $\gamma$, CCL-2, and IL-1 $\beta$ ) levels in serum obtained from mice or culture medium were measured using enzyme-linked immunosorbent assay kits (R\&D Systems, Minneapolis, MN, USA).

\section{Statistical analysis}

Data are expressed as the mean \pm standard error of the mean (s.e.m.) or standard deviation (s.d.). The statistical significance of differences between two conditions was estimated using an unpaired $t$-test. One-way ANOVA was used to compare multiple (more than two) conditions. Tukey's post hoc test was used for multiple comparisons. A $p$-value $<0.05$ was deemed significant. The variance was similar between groups that were being statistically compared. All statistical analyses were performed using GraphPad Prism 8.2 software (GraphPad, San Jose, CA, USA). No data were excluded from the analyses.

\section{Acknowledgements}

We thank S. Lee and Prof. W.U. Kim for advice to establish experimental techniques. This research was funded by the National Research Foundation grants (NRF-2018R1A5A1024340, NRF-2017R1E1A1A01074673, NRF2016R1D1A1B03932335, and NRF-2019R1A2C1089260) and Health Technology R\&D Project grant (H16C1837) of Korea. This work was also supported by UNIST funds (1.180081.01).

\section{Conflict of interest}

The authors declare that they have no conflict of interest.

\section{Publisher's note}

Springer Nature remains neutral with regard to jurisdictional claims in published maps and institutional affiliations.

Supplementary Information accompanies this paper at (https://doi.org/ 10.1038/s41419-020-2632-8).

Received: 9 December 2019 Revised: 19 May 2020 Accepted: 21 May 2020 Published online: 04 June 2020

\section{References}

1. Shortman, K. \& Naik, S. H. Steady-state and inflammatory dendritic-cell development. Nat. Rev. Immunol. 7, 19-30 (2007).

2. Dalod, M., Chelbi, R., Malissen, B. \& Lawrence, T. Dendritic cell maturation: functional specialization through signaling specificity and transcriptional programming. EMBO 33, 1104-1116 (2014).

3. Ganguly, D., Haak, S., Sisirak, V. \& Reizis, B. The role of dendritic cells in autoimmunity. Nat. Rev. Immunol. 13, 566-577 (2013).
4. Gibofsky, A. Overview of epidemiology, pathophysiology, and diagnosis of rheumatoid arthritis. Am. J. Manag. Care 18, S295-S302 (2012).

5. Combe, B. et al. Eular recommendations for the management of early arthritis: report of a task force of the European Standing Committee for International Clinical Studies Including Therapeutics (ESCISIT). Ann. Rheum. Dis. 66, 34-45 (2006).

6. Smolen, J. S., Aletaha, D., Koeller, M., Weisman, M. \& Emery, P. New therapies for the treatment of rheumatoid arthritis. Lancet 370, 1861-1874 (2007).

7. Smolen, J. S. et al. Treating rheumatoid arthritis to target: 2014 update of the recommendations of an international task force. Ann. Rheum. Dis. 75, 3-15 (2016).

8. Smolen, J. S., Aletaha, D. \& McInnes, I. B. Rheumatoid arthritis. Lancet $\mathbf{3 8 8}$, 2023-2038 (2016)

9. Thomas, R. \& Quinn, C. Functional differentiation of dendritic cells in rheumatoid arthritis: role of CD86 in the synovium. J. Immunol. 156, 3074-3086 (1996).

10. McInnes, I. B. \& Schett, G. The pathogenesis of rheumatoid arthritis. N. Engl. J. Med. 365, 2205-2219 (2011).

11. Lebre, M. C. et al. Rheumatoid arthritis synovium contains two subsets of CD83-DC-LAMP-dendritic cells with distinct cytokine profiles. Am. J. Pathol. 172, 940-950 (2008)

12. Ramwadhdoebe, T. H. et al. CD1C+ dendritic cells are overrepresented in lymph nodes of early arthritis patients and related to B cell response. Ann. Rheum. Dis. 73, A70 (2014).

13. Richez, C. et al. Myeloid dendritic cells correlate with clinical response whereas plasmacytoid dendritic cells impact autoantibody development in rheumatoid arthritis patients treated with infliximab. Arthritis Res. Ther. 11, R100 (2009).

14. Thomas, R., Davis, L. S. \& Lipsky, P. E. Rheumatoid synovium is enriched in mature antigen-presenting dendritic cells. J. Immunol. 152, 2613-2623 (1994).

15. Jongbloed, S. L. et al. Enumeration and phenotypical analysis of distinct dendritic cell subsets in psoriatic arthritis and rheumatoid arthritis. Arthritis Res. Ther. 8, R15 (2006).

16. Miyakawa, H., Woo, S. K., Dahl, S. C., Handler, J. S. \& Kwon, H. M. Tonicity responsive enhancer binding protein, a rel-like protein that stimulates transcription in response to hypertonicity. Proc. Natl Acad. Sci. USA 96, 2538-2542 (1999).

17. Lopez-Rodriguez, C., Aramburu, J., Rakeman, A. S. \& Rao, A. NFAT5, a constitutively nuclear NFAT protein that does not cooperate with Fos and Jun. Proc. Natl Acad. Sci. USA 96, 7214-7219 (1999).

18. Go, W. Y., Liu, X., Roti, M. A., Liu, F. \& Ho, S. N. NFAT5/TonEBP mutant mice define osmotic stress as a critical feature of the lymphoid microenvironment Proc. Natl Acad. Sci. USA 101, 10673-10678 (2004).

19. Lee, S. D. et al. TonEBP stimulates multiple cellular pathways for adaptation to hypertonic stress: organic osmolyte-dependent and -independent pathways. Am. J. Physiol. Ren. Physiol. 300, F707-F715 (2011).

20. Lee, N., Kim, D. \& Kim, W. U. Role of NFAT5 in the immune system and pathogenesis of autoimmune diseases. Front. Immunol. 10, 270 (2019).

21. Aramburu, J. \& López-Rodríguez, C. Regulation of inflammatory functions of macrophages and T lymphocytes by NFAT5. Front. Immunol. 10, 535 (2019).

22. Kleinewietfeld, $M$. et al. Sodium chloride drives autoimmune disease by the induction of pathogenic TH17 cells. Nature 496, 518-522 (2013).

23. Buxade, M. et al. Gene expression induced by Toll-like receptors in macrophages requires the transcription factor NFAT5. J. Exp. Med. 209, 379-393 (2012).

24. Lee, H. H. et al. LPS-induced NFKB enhanceosome requires TonEBP/NFAT5 without DNA binding. Sci. Rep. 6, 24921 (2016).

25. Choi, S. Y. et al. TonEBP suppresses IL-10-mediated immunomodulation. Sci. Rep. 6, 25726 (2016).

26. Halterman, J. A., Kwon, H. M., Leitinger, N. \& Wamhoff, B. R. NFAT5 expression in bone marrow-derived cells enhances atherosclerosis and drives macrophage migration. Front. Physiol. 3, 1-7 (2012).

27. Choi, S. et al. Transcription factor NFAT5 promotes macrophage sunvival in rheumatoid arthritis. J. Clin. Invest. 127, 954-969 (2017).

28. Choi, S. Y. et al. Tonicity-responsive enhancer-binding protein mediates hyperglycemia-induced inflammation and vascular and renal injury. J. Am. Soc. Nephrol. 29, 492-504 (2018).

29. Lee, J. H. et al. Tonicity-responsive enhancer-binding protein promotes hepatocellular carcinogenesis, recurrence and metastasis. Gut 68, 347-358 (2019). 
30. Yoon, H. J. et al. NFAT5 is a critical regulator of inflammatory arthritis. Arthritis Rheum. 63, 1843-1852 (2011).

31. Köttgen, A. et al. New loci associated with kidney function and chronic kidney disease. Nat. Genet. 42, 376-384 (2010).

32. Mahajan, A. et al. Fine-mapping type 2 diabetes loci to single-variant resolution using high-density imputation and islet-specific epigenomic maps. Nat. Genet. 50, 1505-1513 (2018).

33. Choi, S. Y., Lee-Kwon, W. \& Kwon, H. M. The evolving role of TonEBP as an immunometabolic stress protein. Nat. Rev. Nephrol. https://doi.org/10.1038/ s41581-020-0261-1 (2020).

34. Schulze-Koops, H. \& Kalden, J. R. The balance of Th1/Th2 cytokines in rheumatoid arthritis. Best Pract. Res. Clin. Rheumatol. 15, 677-691 (2001).

35. Azizi, G., Jadidi-Niaragh, F. \& Mirshafiey, A. Th17 Cells in immunopathogenesis and treatment of rheumatoid arthritis. Int. J. Rheum. Dis. 16, 243-253 (2013).

36. Chyou, $\mathrm{S}$. et al. Coordinated regulation of lymph node vascular-stromal growth first by CD11c+ cells and then by T and B cells. J. Immunol. 187, 5558-5567 (2011).

37. Yang, C. Y. et al. Trapping of naive lymphocytes triggers rapid growth and remodeling of the fibroblast network in reactive murine lymph nodes. Proc. Natl Acad. Sci. USA 111, 109-118 (2014).

38. Lindquist, R. L. et al. Visualizing dendritic cell networks in vivo. Nat. Immunol. 5, 1243-1250 (2004).

39. Wenink, M. H., Han, W., Toes, R. E. \& Radstake, T. R. Dendritic cells and their potential implication in pathology and treatment of rheumatoid arthritis. Handb. Exp. Pharm. 188, 81-98 (2009).

40. Kellermann, S. A., Hudak, S., Oldham, E. R., Liu, Y. J. \& McEvoy, L. M. The CC chemokine receptor-7 ligands 6 Ckine and macrophage inflammatory protein3 beta are potent chemoattractants for in vitro- and in vivo-derived dendritic cells. J. Immunol. 162, 3859-3864 (1999).

41. Riol-Blanco, L. et al. The chemokine receptor CCR7 stimulates two signaling pathways that independently modulate chemotaxis and migratory speed of dendritic cells. J. Immunol. 174, 4070-4080 (2005).

42. Helft, J. et al. GM-CSF mouse bone marrow cultures comprise a heterogeneous population of $\mathrm{CD} 11 \mathrm{C}(+) \mathrm{MHCll}(+)$ macrophages and dendritic cells. Immunity 42, 1197-1211 (2015).

43. Na, Y. R., Jung, D., Gu, G. J. \& Seok, S. H. GM-CSF grown bone marrow derived cells are composed of phenotypically different dendritic cells and macrophages. Mol. Cells 39, 734-741 (2016).

44. Buxadé, $M$. et al. Macrophage-specific MHCll expression is regulated by a remote Ciita enhancer controlled by NFAT5. J. Exp. Med. 215, 2901-2918 (2018).

45. Iwasaki, A. \& Medzhitov, R. Toll-like receptor control of the adaptive immune responses. Nat. Immunol. 5, 987-995 (2004).

46. Rescigno, M., Martino, M., Sutherland, C. L., Gold, M. R. \& Ricciardi-Castagnoli, P. Dendritic cell survival and maturation are regulated by different signaling pathways. J. Exp. Med. 188, 2175-2180 (1998).
47. Ardeshna, K. M., Pizzey, A. R., Devereux, S. \& Khwaja, A. The PI3 kinase, p38 SAP kinase, and NF-kappaB signal transduction pathways are involved in the survival and maturation of lipopolysaccharide-stimulated human monocytederived dendritic cells. Blood 96, 1039-1046 (2000).

48. Baldwin, H. M., Ito-lhara, T., Isaacs, J. D. \& Hilkens, C. M. Tumour necrosis factor alpha blockade impairs dendritic cell survival and function in rheumatoid arthritis. Ann. Rheum. Dis. 69, 1200-1207 (2010).

49. Mascanfroni, I. D. et al. IL-27 acts on DCs to suppress the T cell response and autoimmunity by inducing expression of the immunoregulatory molecule CD39. Nat. Immunol. 14, 1054-1063 (2013).

50. Yen, J. H., Kong, W. \& Ganea, D. IFN-beta inhibits dendritic cell migration through STAT-1-mediated transcriptional suppression of CCR7 and matrix metalloproteinase 9. J. Immunol. 184, 3478-3486 (2010).

51. Audiger, C., Rahman, M. J., Yun, T. J., Tarbell, K. V. \& Lesage, S. The importance of dendritic cells in maintaining immune tolerance. J. Immunol. 198 2223-2231 (2017).

52. Cuda, C. M., Pope, R. M. \& Perlman, H. The inflammatory role of phagocyte apoptotic pathways in rheumatic diseases. Nat. Rev. Rheumatol. 12, 543-558 (2016).

53. Alivernini, S. et al. Driving chronicity in rheumatoid arthritis: perpetuating role of myeloid cells. Clin. Exp. Immunol. 193, 13-23 (2018).

54. Guilliams, M. et al. Dendritic cells, monocytes and macrophages: a unified nomenclature based on ontogeny. Nat. Rev. Immunol. 14, 571-578 (2014)

55. Ma, W. T., Gao, F., Gu, K. \& Chen, D. K. The role of monocytes and macrophages in autoimmune diseases: a comprehensive review. Front. Immunol. 10, 1140 (2019).

56. Roberts, C. A., Dickinson, A. K. \& Taams, L. S. The interplay between monocytes/macrophages and $\mathrm{CD}^{+} \mathrm{T}$ cell subsets in rheumatoid arthritis. Front. Immunol. 6, 571 (2015).

57. Küper, C., Beck, F. X. \& Neuhofer, W. Generation of a conditional knockout allele for the NFAT5 gene in mice. Front. Physiol. 5, 507 (2015).

58. Kim, W. U. et al. Suppression of collagen-induced arthritis by single administration of poly (lactic-Co-glycolic acid) nanoparticles entrapping type II collagen: a novel treatment strategy for induction of oral tolerance. Arthritis Rheum. 46, 1109-1120 (2002).

59. Matthys, P. et al. Anti-IL-12 antibody prevents the development and progression of collagen-induced arthritis in IFN-gamma receptor-deficient mice. Eur. J. Immunol. 28, 2143-2151 (1998).

60. Link, A. et al. Fibroblastic reticular cells in lymph nodes regulate the homeostasis of naive T cells. Nat. Immunol. 8, 1255-1265 (2007).

61. Hochrein, H., Jahrling, F., Kreyschh, H. G. \& Sutter, A. Immunophenotypical and functional characterization of bone marrow derived dendritic cells. Adv. Exp. Med. Biol. 378, 61-63 (1995).

62. Weischenfeldt, J. \& Porse, B. Bone marrow-derived macrophages (BMM): isolation and applications. CSH Protoc. 1, 2008 (2008). 